\title{
THE CONIC AS A SPACE ELEMENT*
}

\author{
BY
}

\author{
ROGER A. JOHNSON
}

\section{INTRODUCTION}

In this article it is proposed to determine a system of coördinates for the conic in space of three dimensions, analogous to the line coördinates of Plücker, and the similar systems for the circle in two and in three dimensions; and to study systems of conics by means of these coördinates.

The conic in space has been considerably studied in the last sixty years, but no systematic theory has been developed. In 1908, the Belgian Royal Academy announced the offer of a prize for a discussion of the subject, but so far as the writer knows, no award was made.

It will be of interest to summarize briefly the work that has been done in this field.

In 1861 Grunert $\uparrow$ treated the general theory of the conic as a space curve, in connection with the study of planetary orbits. Defining a conic by choosing arbitrarily a line as directrix, a point as focus, and the eccentricity, he deduces a number of analytic relations. He also treats a number of other problems, chiefly of interest to the astronomer.

About the same time Chasles $\ddagger$ laid the foundations of a theory of characteristics of conics in space, analogous to the well-known theory for conics in the plane. Hierholzer§ and Lüroth\| discuss similar problems.

The earliest attempt to determine a set of coördinates for a conic is doubtless that of Spottiswoode. T His coördinates are not the simplest nor the most natural ones, however. We shall touch on them later $(\S 3,6$, footnote).

A treatment somewhat analogous to parts of ours is given by $\mathrm{P}$. van Geer.** His methods, however, are clumsy, and such of his results as are of value we can obtain by more direct methods.

\footnotetext{
* Presented to the Society, September 8, 1914.

† Grunert's Archiv, 1861, pp. 1-104.

$\ddagger$ Comptes Rendus, 1865, pp. 389-397.

$\S$ Mathematische Annalen, vol. 2 (1870), pp. 563-580.

$\|$ M athematische Annalen, vol. 3 (1871), pp. 124-133.

IProceedings of the London Mathematical Society, 1878, pp. 185-196,

** A r chives Né erlandaises, 1888 , pp. 58-90.

Trans. Am. Math. Soc. 23
} 
Some parts of this paper are based on an article by Reye* on linear systems of quadrics. It will turn out most desirable for us to regard a conic as a special quadric envelope, and hence the methods of studying systems of quadrics are applicable to the study of conics. In the article in question, Reye does not give much special attention to systems of conics; but from his results we can derive at once considerable information concerning linear systems of conics.

For the most part, however, systems of conics have been treated by pure geometrical methods. Several writers have studied particular systems, each defined in a special way. For example, Humbert $\dagger$ discusses the following system: through five fixed points there are $\infty^{2}$ cubic curves; of the $\infty^{1}$ that touch a fixed plane, the points of contact in general lie on a conic. Thus, a conic is determined in every plane of space; and the paper is a very interesting study of this system. The methods, however, are applicable only to this particular system, and the results no more general than this system. The same remarks apply to the papers of Pieri, $\ddagger$ de Vries, $\S$ and the first article of Montesano.\|

Since 1890, however, there has been developed a general theory of two and three parameter systems of certain types, the authors being Prof. Domenico Montesano of Pisa and M. Lucien Godeaux of Liège. The former T has given a complete treatment of linear congruences,-that is, of two-parameter families with one member through an arbitrary point. In a paper read before the Rome Congress, ** he sketched the theory of "bilinear" complexes or three parameter families.

Godeaux, in a number of short notes, $\dagger \dagger$ has considered the same problems. In a later note, $¥$ he called attention to some alleged errors in Montesano's work, and offered corrections. This elicited from Prof. Montesano a rather spirited reply $\S$ showing deficiencies in the reasoning of M. Godeaux. Apparently they are in agreement as to the facts of the case.

We shall not consider these matters further, for in the present paper we shall adopt a different point of view. Namely, these writers regard a conic as a curve of order 2, while we shall mean by a conic a degenerate envelope of class 2. Hence our totality of conics is a different entity from theirs, and in

\footnotetext{
*Crelle, Journal für Mathematik, vol. 82 (1876), pp. 54-83.

†Journal de l' école polytechnique, vol. 62 (1894), pp. 123-149.

† Torino Atti, vol. 28, pp. 289-303.

§ A m sterdam A ka demie, Verslagen, 1904, pp. 281-284.

\|Rendicontidel Reale Istituto Lombardo, 1893, pp. 589-604.

T $N$ a poli Rendic ont i, 1895-6, pp. 155-181.

${ }^{* *}$ Atti del IV Congresso Internazionale, Roma, 1908, II, pp. 231-

††Bulletin de l'Académie de Belgique, 1908-1910.

łł $\mathrm{P}$ aris Comptes Rendus, vol. 152 (1911), pp. 1149-1151.

$\S \S$ Paris Comptes Rendus, vol. 153 (1911), pp. 45-48.
} 233. 
dealing with systems of conics, which in general include degenerate members, the results will be different.

In addition to his work on congruences and complexes Godeaux* has at tempted to devise coördinates for the conic in space. His most successfu method is that of defining a conic as the intersection of a plane with a quadric linearly dependent on six fixed quadrics. The coördinates of the conic are then the four coördinates of the plane and the six multipliers of the system of quadrics. He makes some use of these coördinates in studying linear systems, but to us they seem to have little value. He suggests several other schemes, but they appear to be of even less use than the one we have discussed.

\section{Determination of Coördinates}

In studying conics in space, it is desirable to determine a system of coördinates, such that to a conic there always corresponds a unique set of coördinates, and conversely.

Two simple methods of defining a conic suggest themselves.

(a) If $\left(u_{1}, u_{2}, u_{3}, u_{4}\right)$ be homogeneous coördinates of a plane, the equation

$$
\sum_{i, j=1}^{4} a_{i j} u_{i} u_{j}=0
$$$$
\left(a_{i j}=a_{j i}\right)
$$

represents an envelope of class two, which, when the matrix

$$
\left\|\begin{array}{llll}
a_{11} & a_{12} & a_{13} & a_{14} \\
a_{21} & a_{22} & a_{23} & a_{24} \\
a_{31} & a_{32} & a_{33} & a_{34} \\
a_{41} & a_{42} & a_{43} & a_{44}
\end{array}\right\|
$$

is of rank three, is a conic. Conversely, any non-degenerate conic of space may be represented by an equation of the type (1) with vanishing discriminant.

This suggests the possibility of taking as coördinates of a conic a set of ten homogeneous numbers

$$
\left(a_{11}, a_{12}, \cdots, a_{44}\right) \quad\left(a_{i j}=a_{j i}\right),
$$

which satisfy the quartic identity

$$
\left|\begin{array}{cccc}
a_{11} & a_{12} & a_{13} & a_{14} \\
a_{21} & a_{22} & a_{23} & a_{24} \\
a_{31} & a_{32} & a_{33} & a_{34} \\
a_{41} & a_{42} & a_{43} & a_{44}
\end{array}\right|=0 .
$$

*Bulletin de l'Académie Belgique, 1908, pp, 896-902. 
For we have established a one-to-one correspondence between non-degenerate conics and those sets (a) whose matrix is of rank three.

(b) The second method of representing a conic is the parametric form

$$
x_{i}=a_{i} s^{2}+2 b_{i} s t+c_{i} t^{2} \quad(i=1, \cdots, 4),
$$

where the $a$ 's, $b$ 's, and $c$ 's are constants, and $(s, t)$ are homogeneous parameters. Any set of twelve numbers $(a, b, c)$ define a conic uniquely, in general; but the converse is not true, since these coefficients may be changed by a linear transformation of the parameters which does not change the conic.

The last remark suggests that we may get a desirable set of coördinates by choosing functions of these coefficients that are invariant under all proper linear transformations of the parameters. The simplest invariants of the forms

are the expressions

$$
a_{i} s^{2}+2 b_{i} s t+c_{i} t^{2} \quad(i=1, \cdots, 4),
$$

$$
w_{i j}=\frac{1}{2}\left(a_{i} c_{j}+c_{i} a_{j}-2 b_{i} b_{j}\right)
$$

whereof there are sixteen, reducing to ten by virtue of the obvious relation $w_{i j}=w_{j i}$. We have then, for any conic given by (5), a homogeneous set of numbers (7). Clearly they cannot be independent coördinates of a conic; for since the conics of space form an eight-parameter family, we have one superfluous coördinate.

Let us see how the numbers $(w)$ are related to the coördinates indicated under $(a)$. We will find the condition that the conic (5) touches an arbitrary plane

$$
(v x) \equiv v_{1} x_{1}+v_{2} x_{2}+v_{3} x_{3}+v_{4} x_{4}=0 .
$$

The two points in which the conic cuts the plane are given by the equation

$$
(v a) s^{2}+2(v b) s t+(v c) t^{2}=0 .
$$

These points coincide, and the plane touches the conic, if and only if

$$
\left|\begin{array}{ll}
(v a) & (v b) \\
(v b) & (v c)
\end{array}\right|=0 .
$$

Expanding this equation, we have by means of (7)

$$
\sum_{i, j=1}^{4} w_{i j} v_{i} v_{j}=0,
$$

and we see that the ten numbers $w_{i j}$ are precisely the same coördinates to which the first treatment led us. Thus it appears that they are very desirable coördinates for a conic in space.

We next wish to extend our notion of conic in such a way that the correspondence between conics and sets of coördinates shall be without exception. Accordingly, we formulate the following 
Definition. Let

$$
\left\|\begin{array}{llll}
w_{11} & w_{12} & w_{13} & w_{14} \\
w_{21} & w_{22} & w_{23} & w_{24} \\
w_{31} & w_{32} & w_{33} & w_{34} \\
w_{41} & w_{42} & w_{43} & w_{44}
\end{array}\right\|
$$

$$
\left(w_{i i}=w_{i j}\right)
$$

be a set of numbers not all zero, satisfying the relation

$$
\left|\begin{array}{llll}
w_{11} & w_{12} & w_{13} & w_{14} \\
w_{21} & w_{22} & w_{23} & w_{24} \\
w_{31} & w_{32} & w_{33} & w_{34} \\
w_{41} & w_{42} & w_{43} & w_{44}
\end{array}\right|=0
$$

Then the envelope of class 2 , defined by the equation $\Sigma w_{i j} u_{i} u_{j}=0$ shall be called $a$ conic.

Obviously conics are to be classified according to the rank of the matrix $(w)$.

Case 1. $(w)$ is of rank 3. Then the envelope is a non-degenerate plane conic, or, as we shall say, a proper conic.

Case 2. ( $w$ ) is of rank 2. In this case the left-hand side of equation (12) is reducible into two linear factors, and the planes satisfying (12) are the totality of planes passing through either of two fixed points. Hence the envelope consists of these two points, and we shall call such a conic a point-pair.

Case 3. $(w)$ is of rank 1. Then the left-hand side of (12) is the square of a linear form, and the envelope is a single point counted twice. Such a conic we shall call a point-double.

From this definition it follows that there is a complete one-to-one correspondence between conics of space and sets of coördinates $w$ with vanishing determinant. But sets of $n+1$ homogeneous coördinates can always be put in one-to-one correspondence with points of a projective linear space of $n$ dimensions. Hence we have the following representation of conics:

Since a quadric envelope is determined by ten homogeneous coördinates $w_{i j}$, the totality of such quadrics correspond in a one-to-one manner with points of a linear space of nine dimensions $S_{9}$. In this domain the conics of space are represented by points of an eight-dimensional hyperspace of order four, whose equation is given by (12). We shall denote this spread $S_{8}^{4}$ by $\Sigma$.

\section{Elementary Properties}

1. Consider a conic $(w)$ of rank 3 . Write

$$
\Delta(w) \equiv\left|\begin{array}{llll}
w_{11} & w_{12} & w_{13} & w_{14} \\
w_{21} & w_{22} & w_{23} & w_{24} \\
w_{31} & w_{32} & w_{33} & w_{34} \\
w_{41} & w_{42} & w_{43} & w_{44}
\end{array}\right|=0
$$


We shall denote the co-factor of any element $w_{i j}$ of this determinant by $W_{i j}$. Then by hypothesis the $W$ 's do not all vanish, and

$$
\Delta \equiv \sum_{i=1}^{4} w_{i j} W_{i j} \equiv \sum_{i=1}^{4} w_{i j} W_{i j}
$$

Theorem. The adjoint matrix

is of rank unity.

$$
\left\|\begin{array}{llll}
W_{11} & W_{12} & W_{13} & W_{14} \\
W_{21} & W_{22} & W_{23} & W_{24} \\
W_{31} & W_{32} & W_{33} & W_{34} \\
W_{41} & W_{42} & W_{43} & W_{44}
\end{array}\right\|
$$

For at least one member is not zero, by hypothesis; but every two-rowed determinant of (3) has $\Delta$ as a factor, and hence vanishes.

It follows that the $W$ 's are dependent on four parameters; and in fact, if we set

(4) $\quad W_{11}=D_{1}^{2}, \quad W_{12}=D_{1} D_{2}, \quad W_{13}=D_{1} D_{3}, \quad W_{14}=D_{1} D_{4}$ we obtain

$$
W_{i j}=D_{i} D_{j} \quad(i, j=1, \cdots, 4) .
$$

We can show at once that the $D$ 's so defined are the coördinates of the plane of the conic. For in general the quadric

$$
\sum_{i, j=1}^{4} w_{i j} u_{i} u_{j}=0
$$

has one and only one singular plane, if the system

$$
\begin{aligned}
& w_{11} u_{1}+w_{12} u_{2}+w_{13} u_{3}+w_{14} u_{4}=0, \\
& w_{21} u_{1}+w_{22} u_{2}+w_{23} u_{3}+w_{24} u_{4}=0, \\
& w_{31} u_{1}+w_{32} u_{2}+w_{33} u_{3}+w_{34} u_{4}=0, \\
& w_{41} u_{1}+w_{42} u_{2}+w_{43} u_{3}+w_{44} u_{4}=0,
\end{aligned}
$$

has one and essentially only one solution not consisting of zeros; i. e., if the matrix $(w)$ is of rank 3 . When this occurs, there are three of the equations (7) that are linearly independent; and by solving them we get the coördinates of the plane. For example, if $W_{11} \neq 0$, so that the last three equations are independent, we have from these three

$$
u_{1}: u_{2}: u_{3}: u_{4}=W_{11}: W_{12}: W_{13}: W_{14}=D_{1}: D_{2}: D_{3}: D_{4},
$$

as was to be shown.

2. Consider next a conic $(w)$ of rank 2. In this case, the equations (7) 
are equivalent to two equations; and therefore the system has $\infty^{1}$ solutions, linearly dependent on two. That is to say, a conic of rank two has a pencil of singular planes.

We have noted that in this case the envelope consists of two points; and it is obvious that the singular planes are the planes through both of these points. Hence the axis of the pencil is the line joining the two points. We shall call this line the axis of the conic. Its coördinates may be found as follows.

Each of the equations (7), in which not all the coefficients vanish, is the equation of a point, whose coördinates are the coefficients of the $u$ 's. Further, since every singular plane satisfies (7), these points lie on the axis of the conic. For definiteness assume that

$$
\left|\begin{array}{ll}
w_{11} & w_{12} \\
w_{21} & w_{22}
\end{array}\right| \neq 0
$$

Then $\left(w_{11}, w_{12}, w_{13}, w_{14}\right)$ and $\left(w_{21}, w_{22}, w_{23}, w_{24}\right)$ are coördinates of two distinct points on the axis, and the plücker coördinates of the latter are accordingly given by

$$
\rho_{12} p_{i j}=\left|\begin{array}{ll}
w_{1 i} & w_{1 j} \\
w_{2 i} & w_{2 j}
\end{array}\right| \quad(i=1, \cdots, 4),
$$

where $\rho_{12}$ is a constant factor not zero.

Similarly, for any two distinct equations of (7),

$$
\rho_{m n} p_{i j}=\left|\begin{array}{cc}
w_{m i} & w_{m j} \\
w_{n i} & w_{n j}
\end{array}\right| \quad(i, j=1, \cdots, 4) \text {. }
$$

To determine $\rho_{m n}$, set $i=1, j=2$. Then

$$
\rho_{m n} p_{12}=\left|\begin{array}{ll}
w_{m 1} & w_{m 2} \\
w_{n 1} & w_{n 2}
\end{array}\right| \equiv\left|\begin{array}{ll}
w_{1 m} & w_{1 n} \\
w_{2 m} & w_{2 n}
\end{array}\right|=\rho_{12} p_{m n},
$$

whence if we take $\rho_{12}=p_{12}$, we have $\rho_{m n}=p_{m n}$.

Obviously also when $p_{m n}=0$, then $\rho_{m n}=0$ also; and we have the

Theorem. Let $(w)$ be a conic of rank two, and let $(p)$ be the plücker coördinates of its axis. Then

$$
p_{i j} p_{m n}=\left|\begin{array}{ll}
w_{i m} & w_{i n} \\
w_{j m} & w_{j n}
\end{array}\right| \text {. }
$$

3. Consider a conic $(w)$ of rank 1 . We have

$$
\sum_{i, j=1}^{4} w_{i j} u_{i} u_{j} \equiv\left(x_{1} u_{1}+x_{2} u_{2}+x_{3} u_{3}+x_{4} u_{4}\right)^{2}
$$

and hence

$$
w_{i j}=x_{i} x_{j}
$$


Hence when a conic is a point-double, we can at once write the coördinates of its point; and conversely, we have at once the $w$-coördinates of the pointdouble at any point.

\section{Relations to Point, Line, and Plane}

It is now proposed to determine the representation in $S_{9}$ of simple systems of conics in space.

1. First let us recall that a set of coördinates $(w)$ define on the one hand a quadric envelope, and on the other hand a point of a nine dimensional space $S_{9}$.

If, in particular,

$$
\Delta(w)=0,
$$

the envelope is a conic, and the corresponding point lies on a quartic hypersurface which we shall call $\Sigma$.

2. The condition that a conic be tangent to a plane $(u)$ is obviously

$$
\sum_{i, j=1}^{4} w_{i j} u_{i} u_{j}=0 .
$$

When $(u)$ is given, this is linear in $(w)$. Hence we have the result:

The totality of conics that touch a fixed plane $(u)$ are represented in $S_{9}$ by the intersection of $\Sigma$ with a hyperplane whose equation is (2).

Evidently there are $\infty^{3}$ such hyperplanes. We will now show that they are precisely the tangent hyperplanes to $\Sigma$. For the equation of $\Sigma$ is $\Delta(w)=0$, and the polar hyperplane, with regard to $\Sigma$, of any point $(\bar{w})$ is

and this may be written

$$
\sum_{i, j=1}^{4} \frac{\partial \Delta(\bar{w})}{\partial \bar{w}_{\imath j}} w_{i j}=0 ;
$$

$$
\sum_{i, j=1}^{4} \bar{W}_{i j} w_{i j}=0 .
$$

In particular, this hyperplane contains $(\bar{w})$ itself if and only if $\Delta(\bar{w})=0$, and hence $(\bar{w})$ is a point on $\Sigma$. In this case $(\bar{w})$ corresponds to a conic, and the plane of the latter is given by $\bar{W}_{i j}=v_{i} v_{j}$. Hence at any point $(\bar{w})$ of $\Sigma$, the tangent hyperplane is given by

$$
\sum_{i, j=1}^{4} v_{i} v_{j} w_{i j}=0,
$$

where $(v)$ is the plane of the conic $(\bar{w})$. It follows that the hypersurface $\Sigma$ has the same tangent hyperplane at all points which correspond to conics in any given plane; and hence it has only $\infty^{3}$ tangent hyperplanes, each touching it at $\infty^{5}$ points. The $S_{7}^{4}$ (spread of 7 dimensions and order 4) in which a tangent hyperplane cuts $\Sigma$ corresponds to the totality of conics that touch the plane. 
It is clear that these remarks apply only to points of $\Sigma$ that correspond to proper conics; for at other points the tangent hyperplane is indeterminate. That is, the points that correspond to point-pairs and point-doubles generate a singular locus on $\Sigma$; but at ordinary points $\Sigma$ is of a nature analogous to that of a developable surface. To complete the analogy, we observe that the $\infty^{5}$ points of tangency of a tangent hyperplane form a linear spread. For the conditions that the plane $(v)$ be a singular plane of the envelope

$$
\sum_{i, j=1}^{4} w_{i j} u_{i} u_{j}=0
$$

are clearly the four equations

$$
w_{i 1} v_{1}+w_{i 2} v_{2}+w_{i 3} v_{3}+w_{i 4} v_{4}=0 \quad(i=1, \cdots, 4)
$$

If $(v)$ be given, and the $w$ 's be regarded as independent variables except for the conditions $w_{i j}=w_{j i}$, then these equations are independent. From them, however, $\Delta(w)=0$ is a consequence, so that any quadric $(w)$ which satisfies (5) is a conic in the plane $(v)$. Hence the conics in a given plane are the totality of solutions of four linear equations, and so they compose a linear five-parameter family $S_{5}$. Summarizing, we have the

Theorem. The points of $S_{9}$ corresponding to the conics in a given plane form a linear $S_{5}$. Through each ordinary point of $\Sigma$ there passes one and only one such $S_{5}$, which is the totality of points of contact of the tangent hyperplane to $\Sigma$ at that point.

3. In an article on linear systems of quadrics, ${ }^{*}$ Reye deduces a number of results which give us at once several important theorems. He determines the dimension (Stufe) and order (Grad) of the family of conics which satisfy a given condition; from which we have at once the nature of the spread in $S_{9}$ that represents the family. We shall not reproduce his work, which is rather long; but presently we shall summarize his results.

4. We next determine the equation, in point coördinates, of the cone from a point $(y)$ to a conic $(w)$.

First let us find the cone from a fixed point $(y)$ that envelopes a proper quadric $(a)$

$$
\sum_{i, j=1}^{4} a_{i j} u_{i} u_{j}=0 \text {. }
$$

Let $(x)$ be any point, then the condition that a point $\lambda(x)+\mu(y)$ on the line $(y x)$ also lie on the quadric (6) is found by means of the point-equation of the latter,

\footnotetext{
* Loc. cit., Jntroduction.
} 
$(7)\left|\begin{array}{ccccc}0 & \lambda x_{1}+\mu y_{1} & \lambda x_{2}+\mu y_{2} & \lambda x_{3}+\mu y_{3} & \lambda x_{4}+\mu y_{4} \\ \lambda x_{1}+\mu y_{1} & a_{11} & a_{12} & a_{13} & a_{14} \\ \lambda x_{2}+\mu y_{2} & a_{21} & a_{22} & a_{23} & a_{24} \\ \lambda x_{3}+\mu y_{3} & a_{31} & a_{32} & a_{33} & a_{34} \\ \lambda x_{4}+\mu y_{4} & a_{41} & a_{42} & a_{43} & a_{44}\end{array}\right|=0$.

This is quadratic in $\lambda: \mu$. Its roots will be equal and the line will touch the quadric, if and only if

$$
\left|\begin{array}{ccccc}
0 & y_{1} & y_{2} & y_{3} & y_{4} \\
x_{1} & a_{11} & \cdot & \cdot & a_{14} \\
x_{2} & \cdot & \cdot & \cdot & \cdot \\
x_{3} & \cdot & \cdot & \cdot & \cdot \\
x_{4} & a_{41} & \cdot & \cdot & a_{44}
\end{array}\right|-\left|\begin{array}{ccccc}
0 & x_{1} & x_{2} & x_{3} & x_{4} \\
x_{1} & a_{11} & \cdot & \cdot & a_{14} \\
x_{2} & \cdot & \cdot & \cdot & \cdot \\
x_{3} & \cdot & \cdot & \cdot & \cdot \\
x_{4} & a_{41} & \cdot & \cdot & a_{44}
\end{array}\right|\left|\begin{array}{ccccc}
0 & y_{1} & y_{2} & y_{3} & y_{4} \\
y_{1} & a_{11} & \cdot & \cdot & a_{14} \\
y_{2} & \cdot & \cdot & \cdot & \cdot \\
y_{3} & \cdot & \cdot & \cdot & \cdot \\
y_{4} & a_{41} & \cdot & \cdot & a_{44}
\end{array}\right|=0 .
$$

Since $\Delta(a) \neq 0$, this equation is equivalent to the following (cf. Bôcher's Higher Algebra, p. 31).

$$
\left|\begin{array}{llllll}
0 & 0 & x_{1} & x_{2} & x_{3} & x_{4} \\
0 & 0 & y_{1} & y_{2} & y_{3} & y_{4} \\
x_{1} & y_{1} & a_{11} & a_{12} & a_{13} & a_{14} \\
x_{2} & y_{2} & a_{21} & a_{22} & a_{23} & a_{24} \\
x_{3} & y_{3} & a_{31} & a_{32} & a_{33} & a_{34} \\
x_{4} & y_{4} & a_{41} & a_{42} & a_{43} & a_{44}
\end{array}\right|=0,
$$

and this is accordingly the equation of the cone.

If we start with a proper conic instead of a quadric, this method fails. Let, the conic be

$$
\sum_{i, j=1}^{4} w_{i j} u_{i} u_{j}=0, \quad \Delta(w)=0
$$

We form the equation

$$
\sum_{i, j=1}^{4}\left(w_{i j}+\rho y_{i} y_{j}\right) u_{i} u_{j}=0, \quad \rho \neq 0,
$$

which is a quadric through $(w)$ and having the point $(y)$ as vertex of the tangent cone along $(w)$. Setting $a_{i j}=w_{i j}+\rho y_{i} y_{j}$, we have

$$
\Delta(a) \equiv \Delta(w)+\rho \sum_{i, j=1}^{4} y_{i} y_{j} W_{i j} \equiv \rho \sum_{i, j=1}^{4} d_{i} d_{j} y_{i} y_{j} \equiv \rho(d y)^{2}
$$


If $(y)$ is not in the plane $(d)$ of $(w)$, and we take $\rho \neq 0$, then $\Delta(a) \neq 0$, and $(a)$ is a proper quadric. Applying the previous result, we get the desired equation in the form

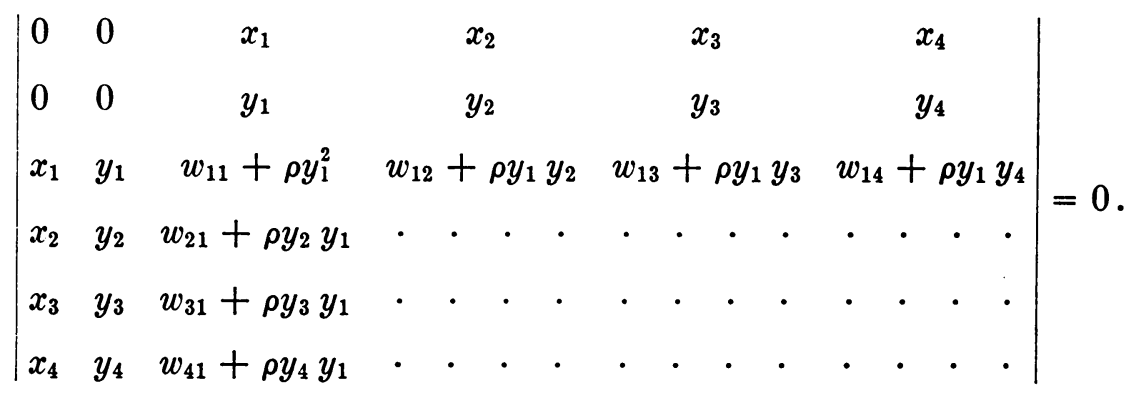

This may be simplified by subtracting multiples of the second row from later rows in an obvious manner. This leads to the

Theorem. The equation of the cone drawn to any proper conic from a point (y) not in its plane is

$$
\left|\begin{array}{cccccc}
0 & 0 & x_{1} & x_{2} & x_{3} & x_{4} \\
0 & 0 & y_{1} & y_{2} & y_{3} & y_{4} \\
x_{1} & y_{1} & w_{11} & \cdot & \cdot & w_{14} \\
x_{2} & y_{2} & \cdot & \cdot & \cdot & \cdot \\
x_{3} & y_{3} & \cdot & \cdot & \cdot & \cdot \\
x_{4} & y_{4} & w_{41} & \cdot & \cdot & w_{44}
\end{array}\right|=0
$$

Remark. It is easily shown that if $(y)$ be a point in the plane of $(w)$, then (14) becomes $(y x)^{2}=0$, where $(y x)=0$ is the equation of the plane.

5. We shall next determine the order of the $S_{6}$ that represents conics through a fixed point.

In the first place, if $(w)$ be a conic whose plane does not pass through $(0,0,0,1)$, then the cone from this point to $(w)$ is given by

$$
\left|\begin{array}{cccc}
0 & x_{1} & x_{2} & x_{3} \\
x_{1} & w_{11} & w_{12} & w_{13} \\
x_{2} & w_{21} & w_{22} & w_{23} \\
x_{3} & w_{31} & w_{32} & w_{33}
\end{array}\right|=0 .
$$

Conversely, if $P:\left(x_{1}, x_{2}, x_{3}, x_{4}\right)$ be a fixed point, the conics $(w)$ that satisfy (15) are those that meet the line from $P_{0}:(0,0,0,1)$ to $P$. For

$(a)$ if $W_{44} \neq 0$, the plane of the conic does not pass through $P_{0}:$ and since $P$ lies on the cone, the element $P_{0} P$ of the cone meets the conic; 
(b) if $W_{44}=0,(15)$ is the equation of the plane of $(w)$; and since both $P$ and $P_{0}$ lie in this plane, their line meets $(w)$ twice.

Now a conic passes through $P$ if and only if

(1) its plane passes through $P$, and (2) it intersects one line through $P$, not lying in its plane.

We shall consider the line $P_{0} P$. The conics that meet it satisfy (15); and this defines in $S_{9}$ a hypersurface $S_{8}^{2}$. The conics whose planes pass through a fixed point $P$ form, as Reye (loc. cit.) shows, a $S_{7}^{6}$. The points common to a $S_{8}^{2}$ and a $S_{7}^{6}$ form a $S_{6}^{12}$. We have just seen that these include two sets of conics: first, the conics through $P$, and second, those whose planes pass through $P_{0} P$. The latter, as Reye (loc. cit.) shows, correspond to a $S_{6}^{4}$. Hence the conics through $P$ are represented by a $S_{6}^{8}$, and we have the

Theonem. The conics which pass through a given point $P$ are represented in $S_{9}$ by $a S_{6}^{8}$.

6. By methods similar to those of part 4 , we can prove the

THEOREM. Let

$$
\sum_{i, j=1}^{4} a_{i j} u_{i} u_{j}=0
$$

be any quadric envelope. Let the plücker coördinates of a line be given by

$$
p_{01}=\pi_{23}, \quad p_{02}=\pi_{31}, \quad p_{03}=\pi_{12}, \quad \text { etc. }
$$

Then the equation

$$
\sum_{1}^{4}\left|\begin{array}{ll}
a_{i j} & a_{i l} \\
a_{k j} & a_{k l}
\end{array}\right| \pi_{i k} \pi_{j l}=0
$$

has the following interpretations:

(a) If (a) is a proper quadric, ( $\pi$ ) touches it.

(b) If ( $a$ ) is a proper conic, $(\pi)$ meets it.

(c) If (a) is a point-pair, $(\pi)$ meets its axis.

(d) If (a) is a point-double, the equation is satisfied identically.

For convenience we make the following

Definition. A line ( $\pi$ ) shall be said to intersect a conic that is a point-pair, when it intersects the axis of the latter.

We have then the

THEOREM. The totality of conics that intersect a given line $(\pi)$ are represented in $S_{9}$ by the total intersection of $\Sigma$ with the $S_{8}^{2}$ whose equation is

$$
\sum_{1}^{4}\left|\begin{array}{ll}
w_{i j} & w_{i l} \\
w_{k j} & w_{k l}
\end{array}\right| \pi_{i k} \pi_{j l}=0 .^{*}
$$

${ }^{*}$ In equation $(16)$, if $(w)$ be regarded as fixed, the equation is that of the complex of lines that meet the conic $(w)$. The coefficients may thus be regarded as coördinates of the conic. This is essentially the system of coördinates used by Spottiswoode. (See Introduction.) 
There are $\infty^{4}$ such $S_{8}^{2}$ 's, corresponding to the lines of space; and they have in common the singular locus (see below) that represents point-doubles.

7. We will close the section with a tabular summary of the correspondence we have developed, including the results obtained by Reye. The latter are indicated by the symbol \#.

In $S_{3}$

Quadric envelope

Conic

Conics in fixed plane

Quadrics touching a fixed plane

Conics whose planes pass through fixed point

Conics whose planes pass through fixed line

Conics which pass through fixed point

Conics that meet a fixed line

Point-pairs

Point-pairs whose lines meet a fixed line

Point-pairs with one member on given line

Point-pairs with both members on given line

Point-pairs with both members in given plane

Point-doubles

Point-doubles in given plane

Point-doubles on given line

$$
\begin{aligned}
& \quad \text { In } S_{9} \\
& \text { Point } \\
& \text { Point of } S_{8}^{4} \equiv \Sigma \\
& S_{5} \text { lying in } \Sigma
\end{aligned}
$$

$S_{8}$. Touching $\Sigma$ at all points of a $S_{5}$ $S_{7}^{6} \#$

$S_{6}^{4} \#$

$S_{6}^{8}(5)$

$S_{7}^{8}(6)$

$S_{6}^{10}$ \#

$S_{5}^{10} \#$

$S_{4}^{4} \#$

$S_{2}^{1} \#$

$S_{4}^{3} \#$

$S_{3}^{8} \#$

$S_{2}^{4} \#$

$S_{1}^{2} \#$

\section{Relations Between Conic and Quadric}

Let us determine the conic in which a given plane cuts a given quadric. We observe that this is exactly dual to a problem already treated $(\S 3,4)$; hence we need not repeat the work. The result is as follows:

THEOREM. The condition that a plane $(u)$ touch the conic $(w)$ in which a given quadric

$$
\sum_{i, j=1}^{4} a_{i j} x_{i} x_{j}=0
$$

is cut by a given plane $\left(A_{1}, A_{2}, A_{3}, A_{4}\right)$ is

$$
\left|\begin{array}{cccccc}
0 & 0 & u_{1} & u_{2} & u_{3} & u_{4} \\
0 & 0 & A_{1} & A_{2} & A_{3} & A_{4} \\
u_{1} & A_{1} & a_{11} & a_{12} & a_{13} & a_{14} \\
u_{2} & A_{2} & a_{21} & a_{22} & a_{23} & a_{24} \\
u_{3} & A_{3} & a_{31} & a_{32} & a_{33} & a_{34} \\
u_{4} & A_{4} & a_{41} & a_{42} & a_{43} & a_{44}
\end{array}\right|=0 .
$$

Hence the coördinates of the conic are immediately written down. If, in particular, the plane $(A)$ touches the quadric, equation (2) reduces to the equation of the point of contact, counted twice. 
We next determine conditions that a conic lie on a given quadric. These conditions do not seem capable of very simple expression. We will treat the problem in two ways. Of these, the former gives a redundant set of conditions; but as usual in such cases, it is impossible to choose a set which will in all cases be sufficient and independent. The second method, on the other hand, gives exactly the right number of equations; but they are complicated in form.

Finst Methiod. If

be a proper quadric, and

$$
\sum_{i, j=1}^{4} A_{i j} u_{i} u_{j}=0
$$

$$
\sum_{i, j=1}^{4} w_{i j} u_{i} u_{j}=0
$$

a conic lying on it, then one member of the pencil linearly dependent on them will be the pole of the plane of $(w)$, counted twice. (This is well known in the theory of quadrics.) Hence $(w)$ lies on $(A)$ if and only if there is a number $k$ such that the matrix

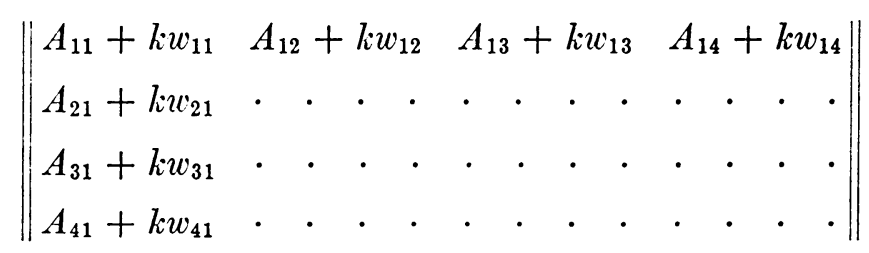

is of rank 1. This gives a number of quadratic equations in $k$, of the type

$$
k^{2}\left|\begin{array}{ll}
w_{11} & w_{12} \\
w_{21} & w_{22}
\end{array}\right|+k\left\{\left|\begin{array}{cc}
w_{11} & A_{12} \\
w_{21} & A_{22}
\end{array}\right|+\left|\begin{array}{ll}
A_{11} & w_{12} \\
A_{12} & w_{22}
\end{array}\right|\right\}+\left|\begin{array}{ll}
A_{11} & A_{12} \\
A_{21} & A_{22}
\end{array}\right|=0 .
$$

The required conditions are that these equations all have a common root, and we may obtain them by equating to zero the resultant of each pair of them. These equations are necessary and sufficient, but are not independent.

SECOND METHOD. This consists in making a non-singular linear transformation that carries the plane of the conic into the plane $(0,0,0,1)$. Let a proper quadric be given by the point equation

$$
\sum_{i, j=1}^{4} a_{i j} x_{i} x_{j}=0, \quad \Delta(a) \neq 0,
$$

and hence by the tangential equation

$$
\sum_{i, j=1}^{4} A_{i j} u_{i} u_{j}=0,
$$


where, as usual, $A_{i j}$ is the cofactor of $a_{i j}$ in $\Delta(a)$. Let $(w)$ be a conic,

$$
\sum_{i, j=1}^{4} w_{i j} u_{i} u_{j}=0, \quad \Delta(w)=0,
$$

and denote the coördinates of its plane by $(d)$. Then

$$
W_{i j}=d_{i} d_{j}, \quad \sum_{i=1}^{4} w_{i j} d_{i}=0 \quad(j=1, \cdots, 4) .
$$

Case 1. $d_{4}=0$. 'Then without making the transformation indicated under case 2, we can carry on the later work directly, as will be obvious.

Case 2. $\quad d_{4} \neq 0$. The linear transformation

$$
\left.\begin{array}{rrr}
u_{1}^{\prime}=d_{4} u_{1} & & -d_{1} u_{4} \\
u_{2}^{\prime}= & d_{4} u_{2} & -d_{2} u_{4} \\
u_{3}^{\prime}= & d_{4} u_{3}-d_{3} u_{4} \\
u_{4}^{\prime}= & & -d_{4} u_{4}
\end{array}\right\}
$$

is non-singular and involutory. We will apply it to the quadratic equations

$$
\begin{aligned}
& \sum_{i, j=1}^{4} w_{i j} u_{i} u_{j}=0 \\
& \sum_{i, j=1}^{4} A_{i j} u_{i} u_{j}=0 .
\end{aligned}
$$

We find that the former goes into

$$
\sum_{i, j=1}^{3} w_{i j} u_{i}^{\prime} u_{j}^{\prime}=0,
$$

while the latter goes into an equation whose matrix is

$$
\left\|\begin{array}{cccc}
d_{4}^{2} A_{11} & d_{4}^{2} A_{12} & d_{4}^{2} A_{13} & -d_{4} \sum_{i=1}^{4} d_{i} A_{i 1} \\
d_{4}^{2} A_{21} & d_{4}^{2} A_{22} & d_{4}^{2} A_{23} & -d_{4} \sum_{i=1}^{4} d_{i} A_{i 2} \\
d_{4}^{2} A_{31} & d_{4}^{2} A_{32} & d_{4}^{2} A_{33} & -d_{4} \sum_{i=1}^{4} d_{i} A_{i 3} \\
-d_{4} \sum_{i=1}^{4} d_{i} A_{i 1} & -d_{4} \sum_{i=1}^{4} d_{i} A_{i 2} & -d_{4} \sum_{i=1}^{4} d_{i} A_{i 3} & +\sum_{i, j=1}^{4} d_{i} d_{j} A_{i j}
\end{array}\right\|,
$$

so that this equation may be written

(15) $d_{4}^{2} \sum_{i, j=1}^{3} A_{i j} u_{i}^{\prime} u_{j}^{\prime}-2 d_{4} \sum_{\substack{i=1 \\ j=1}}^{i=4, j=3} d_{i} A_{i j} u_{j}^{\prime} u_{4}^{\prime}+\left(\sum_{i, j=1}^{4} d_{i} d_{j} A_{i j}\right) u_{4}^{\prime 2}=0$. 
We shall for brevity represent this equation by

$$
\sum_{i, j=1}^{4} k_{i j} u_{i}^{\prime} u_{j}^{\prime}=0 \text {, }
$$

and denoting the cofactors of the elements $k_{i j}$ of (14) by $K_{i j}$, we get the result:

The effect of the transformation (10) on the quadric

is to carry it into the quadric

$$
\sum_{i, j=1}^{4} a_{i j} x_{i} x_{j}=0
$$

$$
\sum_{i, j=1}^{4} K_{i j} x_{i}^{\prime} x_{j}^{\prime}=0 .
$$

We next find the conic in which this quadric cuts the plane $(0,0,0,1)$. In formula (2) set $(A) \equiv(0,0,0,1)$, and $a_{i j} \equiv K_{i j}$. We get

$$
\left|\begin{array}{cccc}
0 & u_{1}^{\prime} & u_{2}^{\prime} & u_{3}^{\prime} \\
u_{1}^{\prime} & K_{11} & K_{12} & K_{13} \\
u_{2}^{\prime} & K_{21} & K_{22} & K_{23} \\
u_{3}^{\prime} & K_{31} & K_{32} & K_{33}
\end{array}\right|=0
$$

as the equation of the conic. But

$$
\left|\begin{array}{ll}
K_{i j} & K_{i m} \\
K_{n j} & K_{n m}
\end{array}\right|=M \Delta(k),
$$

where $M$ is the algebraic complement of $k_{i j} k_{m n}$ in $\Delta(k)$. Hence (17) reduces to

$$
\sum_{i, j=1}^{3}\left|\begin{array}{ll}
k_{i j} & k_{i 4} \\
k_{j 4} & k_{44}
\end{array}\right| u_{i}^{\prime} u_{j}^{\prime}=0,
$$

and this is the conic in which the quadric cuts the plane of the given conic, after the transformation (10).

This coincides with the given conic if and only if

$$
\rho w_{i j}=\left|\begin{array}{ll}
k_{i j} & k_{i 4} \\
k_{j 4} & k_{44}
\end{array}\right| \quad(i, j=1,2,3),
$$

or, when we replace the $k$ 's by their values from (14), and set $d_{i} d_{j}=W_{i j}$,

$$
\rho w_{i j}=\left|\begin{array}{cc}
W_{44} A_{i j} & -\sum_{l=1}^{4} W_{l 4} A_{l j} \\
-\sum_{l=1}^{4} W_{l l} A_{l i} & \sum_{l, m=1}^{4} W_{l m} A_{l m}
\end{array}\right| \quad(i, j=1,2,3) .
$$


These equations are six in number, but they involve an inessential factor $\rho$. By eliminating it, we get exactly the correct number of equations, namely five.

\section{Relations Between Two Conics}

Let $(a)$ and $(b)$ be any two conics. The quadrics linearly dependent on them,

$$
\sum_{j=1}^{4}\left(\lambda a_{i j}+\mu b_{i j}\right) u_{i} u_{j}=0
$$

form a one-parameter family called a series. Among them there are in general four conics, given by values of $\lambda: \mu$ that satisfy the equation

$$
\Delta(\lambda a+\mu b)=0 .
$$

It is known that this equation is invariant under all proper collineations; hence the vanishing of any one of its coefficients is the condition for some special projective re'ation between the two given conics.

We shall represent (2) by the equation

$$
\lambda^{4} \Delta(a)+\lambda^{3} \mu \theta(a, b)+\lambda^{2} \mu^{2} \Phi(a, b)+\lambda \mu^{3} \theta(b, a)+\mu^{4} \Delta(b)=0
$$

and shall refer to the functions $\Theta(a, b), \Theta(b, a), \Phi(a, b)$ as the invariants of the two conics. We observe that the coefficients of $\lambda^{4}$ and $\mu^{4}$ are the discriminants of the conics $(a)$ and $(b)$ respectively, and hence are vanishing invariants. We propose to investigate the meaning of the vanishing of the invariants $\theta$ and $\Phi$.

These expressions are

$$
\begin{gathered}
\Theta(a, b)=\sum_{i, j=1}^{4} b_{i j} A_{i j}, \quad \Theta(b, a)=\sum_{i, j=1}^{4} a_{i j} B_{i j}, \\
\Phi(a, b)=\Phi(b, a)=\sum\left|\begin{array}{llll}
b_{11} & b_{12} & a_{13} & a_{14} \\
b_{21} & b_{22} & a_{23} & a_{24} \\
b_{31} & b_{32} & a_{33} & a_{34} \\
b_{41} & b_{42} & a_{43} & a_{44}
\end{array}\right|,
\end{gathered}
$$

the summation being effected by replacing in turn every pair of columns of $a$ 's by the corresponding $b$ 's, and adding all these determinants.

1. Suppose $\theta(a, b)=0$.

Case a. Let $(a)$ be a proper conic. Denoting the coördinates of its plane by $\left(\alpha_{1}, \alpha_{2}, \alpha_{3}, \alpha_{4}\right)$, we have $A_{i j}=\alpha_{i} \alpha_{j}$. Hence

$$
\Theta(a, b)=\sum_{i, j=1}^{4} b_{i j} A_{i j}=\sum_{i, j=1}^{4} b_{i j} \alpha_{i} \alpha_{j},
$$

Trans. Am. Math. Soc. 24 
and so when $\theta(a, b)$ vanishes, we have

$$
\sum_{i, j=1}^{4} b_{i j} \alpha_{i} \alpha_{j}=0
$$

and $(\alpha)$ is one of the planes that touch the envelope $(b)$.

Case $b$. Let $(a)$ be a degenerate conic. Then $A_{i j}=0(i, j=1, \cdots, 4)$, and for every conic $(b)$ we have $\theta(a, b)=0$.

Remark. It is obvious that the equation

$$
\sum_{i, j=1}^{4} b_{i j} \alpha_{i} \alpha_{j}=0
$$

is satisfied whenever the conics $(a),(b)$ are coplanar.

We have then the result:

Theorem. The equation $\theta(a, b)=0$ is satisfied if and only if one of the following occurs:

(1) The conics (a) and (b) are coplanar; (2) (a) is a proper conic whose plane touches $(b)$; (3) ( $a$ ) is a degenerate conic.

2. If now we interchange $(a)$ and $(b)$, we have the interpretation of $\theta(b, a)$.

3. We next consider the equation

$$
\Phi(a, b)=0 \text {. }
$$

Case $a$. The conics are coplanar. We have already mentioned that the functions $\theta, \Phi$ are invariant under any non-singular linear transformation. If we make such a transformation, carrying the common plane of the conics into the plane $(0,0,0,1)$, their matrices assume the forms

$$
\left[\begin{array}{cccc}
a_{11}^{\prime} & a_{12}^{\prime} & a_{13}^{\prime} & 0 \\
a_{21}^{\prime} & a_{22}^{\prime} & a_{23}^{\prime} & 0 \\
a_{31}^{\prime} & a_{32}^{\prime} & a_{33}^{\prime} & 0 \\
0 & 0 & 0 & 0
\end{array}\right], \quad\left[\begin{array}{cccc}
b_{11}^{\prime} & b_{12}^{\prime} & b_{13}^{\prime} & 0 \\
b_{21}^{\prime} & b_{22}^{\prime} & b_{23}^{\prime} & 0 \\
b_{31}^{\prime} & b_{32}^{\prime} & b_{33}^{\prime} & 0 \\
0 & 0 & 0 & 0
\end{array}\right],
$$

and we see by inspection that $\Phi\left(a^{\prime}, b^{\prime}\right)=0$. Hence the invariant $\Phi$ of two coplanar conics vanishes.

Case b. If either. conic is a point pair, then as we saw in 2, the two-rowed determinants of its matrix are expressible as products of the plücker coördinates of its axis. If $(b)$ is a point-pair, then $\Phi(a, b)=0$ reduces to

$$
\sum_{1}^{4}\left|\begin{array}{ll}
a_{i k} & a_{i l} \\
a_{j k} & a_{j l}
\end{array}\right| \pi_{i j} \pi_{k l}=0,
$$

where the $\pi$ 's are plücker coördinates of the axis of $(b)$. But this $(\S 3)$ is the condition that the line $(\pi)$ meet the conic $(a)$ when the latter is a 
proper conic; and if it is a point-pair, the equation means that the axes of the two point-pairs intersect.

If either conic is a point-double, $\Phi$ vanishes identically.

Case $c$. The remaining case is that neither of the conics is degenerate, and they lie in distinct planes.

To simplify the work, we may without loss of generality take as the planes of the conics the planes $(0,0,0,1)$ and $(1,0,0,0)$ respectively. Then their matrices are

$$
\left[\begin{array}{cccc}
a_{11} & a_{12} & a_{13} & 0 \\
a_{21} & a_{22} & a_{23} & 0 \\
a_{31} & a_{32} & a_{33} & 0 \\
0 & 0 & 0 & 0
\end{array}\right], \quad\left[\begin{array}{cccc}
0 & 0 & 0 & 0 \\
0 & b_{22} & b_{23} & b_{24} \\
0 & b_{32} & b_{33} & b_{34} \\
0 & b_{42} & b_{43} & b_{44}
\end{array}\right]
$$

and $\Phi=0$, reduces to the expression

(10) $\left|\begin{array}{ll}a_{11} & a_{12} \\ a_{21} & a_{22}\end{array}\right| \cdot\left|\begin{array}{ll}b_{33} & b_{34} \\ b_{43} & b_{44}\end{array}\right|-2\left|\begin{array}{cc}a_{11} & a_{13} \\ a_{31} & a_{32}\end{array}\right| \cdot\left|\begin{array}{ll}b_{23} & b_{24} \\ b_{43} & b_{44}\end{array}\right|+\left|\begin{array}{ll}a_{11} & a_{13} \\ a_{31} & a_{33}\end{array}\right|\left|\begin{array}{ll}b_{22} & b_{24} \\ b_{42} & b_{44}\end{array}\right|=0$.

We will determine the pair of points in which the line common to the planes of the conics cuts the conic $(a)$.

The cone from the point $(0,0,0,1)$ to the conic $(a)$ is $(\S 3)$

$$
\left|\begin{array}{cccc}
0 & x_{1} & x_{2} & x_{3} \\
x_{1} & a_{11} & a_{12} & a_{13} \\
x_{2} & a_{21} & a_{22} & a_{23} \\
x_{3} & a_{31} & a_{32} & a_{33}
\end{array}\right|=0
$$

This is cut by the line $x_{1}=0, x_{4}=0$ in the two points found by setting $x_{1}=0$ in this equation. We have

$$
x_{2}^{2}\left|\begin{array}{ll}
a_{11} & a_{13} \\
a_{31} & a_{33}
\end{array}\right|-2 x_{2} x_{3}\left|\begin{array}{ll}
a_{11} & a_{12} \\
a_{31} & a_{32}
\end{array}\right|+x_{3}^{2}\left|\begin{array}{ll}
a_{11} & a_{12} \\
a_{21} & a_{22}
\end{array}\right|=0 .
$$

Similarly, the points in which $(b)$ cuts this line are given by the equation

$$
x_{2}^{2}\left|\begin{array}{ll}
b_{33} & b_{34} \\
b_{43} & b_{44}
\end{array}\right|-2 x_{2} x_{3}\left|\begin{array}{ll}
b_{23} & b_{24} \\
b_{43} & b_{44}
\end{array}\right|+x_{3}^{2}\left|\begin{array}{ll}
b_{22} & b_{24} \\
b_{42} & b_{44}
\end{array}\right|=0
$$

Then the well-known condition that the roots of two quadratic equations separate each other harmonically gives us

by means of equation (10).

$$
\Phi(a, b)=0
$$


Summarizing, we have the

Theorem. Two conics ( $a$ ) and (b) satisfy the relation $\Phi(a, b)=0$ if and only if one of the following occurs:

(1) They are coplanar. (2) They are proper conics in different planes, and separate each other harmonically on the line of intersection of their planes. (3) One of them is a point-pair, and its axis meets the other. (4) They are both pointpairs, with intersecting axes (special case of (1)). (5) One conic is a pointdouble.

4. The condition that two conics intersect at a point is simply expressed in terms of these invariants. Salmon* shows that if two cones have a common tangent plane, the two remaining cones of their pencil fall together.

Dually, if two conics have a common point, the other two conics linearly dependent on them will fall together, and conversely.

Let $(a)$ and $(b)$ be two conics, and let

$$
c_{i j}=\lambda a_{i j}+\mu b_{i j}
$$

The four conics of the series $(c)$ are given by

$$
\Delta(c)=0,
$$

or, since we know that $(a)$ and $(b)$ are two of them, the others are given by

$$
\lambda^{2} \theta(a, b)+\lambda \mu \Phi(a, b)+\mu^{2} \theta(b, a)=0 .
$$

As we have remarked, $(a)$ and $(b)$ intersect if and only if this equation has equal roots, so that we have the result:

Theorem. Two proper conics, $(a)$ and $(b)$, intersect if and only if

$$
\left|\begin{array}{cc}
2 \theta(a, b) & \Phi(a, b) \\
\Phi(a, b) & 2 \Theta(b, a)
\end{array}\right|=0 .
$$

It is seen that this equation is quartic in the coördinates of either conic; hence the conics that meet a fixed conic are represented in $S_{9}$ by a $S_{7}^{16}$, the total intersection of $\Sigma$ with $a S_{8}^{4}$.

\section{Complete and Incomplete Systems-the Incomplete Linear SYSTEMS}

We have seen that every set of numbers $\left(w_{11}, w_{12}, \cdots, w_{44}\right),\left(w_{i j}=w_{j i}\right)$, are the tangential coördinates of a quadric; and in particular, any set that satisfy the relation

$$
\Delta(w)=0
$$

* Geometry of Three Dimensions, 3d ed., § 202. 
are coördinates of a conic. Any set of equations on the $w$ 's, of which $9-r$ are independent, determine an $r$-parameter system of quadrics, and of these, the ones that satisfy (1) are conics. Hence in studying systems of conics defined by equations, we recognize two distinct cases.

Case 1. The equation (1) is not a necessary consequence of the given equations. Then the latter are satisfied by $\infty^{r}$ quadrics, which include $\infty^{r-1}$ conics given by the further equation (1). Such a system of conics we shall call an incomplete system.

Case 2. The equation (1) is satisfied by every solution of the given equations. Then every one of the solutions of the latter is a conic, and the system consists of $\infty^{r}$ conics. A system of this sort we shall call a complete system.

In the nine-dimensional representation of conics and quadrics, a set of $9-r$ independent equations define a spread of dimension $r$. In case this does not lie entirely on $\Sigma$, we have an incomplete system, represented by its intersection with $\Sigma$. In case we have a complete system, the $S_{r}$ lies entirely on $\Sigma$.

Linear systems constitute an important case. The quadrics that satisfy $9-r$ linearly independent linear equations may be given as linearly dependent on $r+1$ quadrics and are represented in $S_{9}$ by a linear $S_{r}$. In general, this will cut $\Sigma$ in a $S_{r-1}^{4}$, and we have an incomplete linear system; but it may happen that the $S_{r}$ lies on $\Sigma$, so that every solution

$$
w_{i j}=\lambda_{1} a_{i j}^{(1)}+\cdots+\lambda_{r+1} a_{i j}^{(r+1)}
$$

satisfies (1), and then we have a complete linear system.

We have already seen the existence of a complete linear system, namely the $\infty^{5}$ conics in a plane. In the following section we shall determine all possible complete linear systems. We take up first incomplete systems.

Evidently it is desirable to study these by means of linear systems of quadrics. The latter have been somewhat studied, and in the simplest cases they are well known. The general one, two and three parameter systems of quadric envelopes are treated in the Encyklopädie, III C2, § VIII, § XII, § XIII. The remaining cases are briefly treated in the same place, $\S$ XIV. The most important work on the subject is that of Reye, in the paper to which we have already referred (Introduction). In this paper he considers linear systems of point-quadrics and of envelope quadrics of all dimensions from one up to eight. From his results, obviously, we can get at once important facts concerning the systems of conics included in his systems of quadrics, though he says little specifically about these conics.

Before considering linear systems, we need to discuss the relation of apolarity, which is closely related. 
Definition. Let

$$
\begin{aligned}
\sum_{i, j=1}^{3} a_{i j} x_{i} x_{j} & =0, \\
\sum_{i, j=1}^{3} b_{i j} u_{i} u_{j} & =0
\end{aligned}
$$

be two conics in a plane. If the relation

$$
\sum_{i, j=1}^{3} a_{i j} b_{i j}=0
$$

is satisfied, the conics are said to be APOLAR.*

The geometrical meaning of the relation is that there exists a triangle selfconjugate with regard to $(a)$ and circumscribed to $(b)$; and dually, a triangle self-conjugate with regard to $(b)$, and inscribed in $(a)$. In either case, when there is one such triangle there are infinitely many.

A line pair or a point pair is apolar to another conic if its members are conjugate with regard to the latter. A line-double is apolar to a conic if the line touches the conic, and a point-double is apolar to a conic if the point lies on the conic.

We next define apolarity of quadrics.

Definition. Two quadrics

$$
\begin{aligned}
& \sum_{i, j=1}^{4} b_{i j} x_{i} x_{j}=0 \\
& \sum_{i, j=1}^{4} a_{i j} u_{i} u_{j}=0
\end{aligned}
$$

that satisfy the relation

$$
\sum_{i, j=1}^{4} a_{i j} b_{i j}=0
$$

are said to be apolar to each other.

Theorem. Two apolar quadrics, neither of which is degenerate, have the property that there exist tetrahedra self-conjugate with regard to the one and inscribed in the other; and dually there exist tetrahedra self-conjugate with regard to the second, and circumscribed about the first. $\dagger$

This proof fails when either quadric is degenerate. We shall next determine

* Various other names are used for this relation. The word "apolar" is due to Reye, who also uses the expressions that $(a)$ supports (stützt) $(b)$, and that $(b)$ rests on (ruht auf) $(a)$. English writers call $(a)$ harmonically inscribed in $(b)$. Rosanes-calls the two conics conjugate.

† For proof see Hesse's Vorlesungen, Kap. 16, p. 190. 
the meaning of apolarity between a proper conic

$$
\sum_{i, j=1}^{4} w_{i j} u_{i} u_{j}=0, \quad \Delta(w)=0,
$$

and an unrestricted point quadric

The relation is

$$
\sum_{i, j=1}^{4} a_{i j} x_{i} x_{j}=0
$$

$$
\sum_{i, j=1}^{4} a_{i j} w_{i j}=0
$$

and this is evidently an invariant for non-singular collineations of space. Hence there is no loss of generality if we take $x_{4}=0$ as the plane of the conic $(w)$. Its equation then becomes

$$
\sum_{i, j=1}^{3} w_{i j} u_{i} u_{j}=0
$$

and the relation of apolarity is

$$
\sum_{i, j=1}^{3} a_{i j} w_{i j}=0,
$$

whence the

THEOREM. The conic (9) is apolar to the quadric (10) if and only if it is apolar to the conic in which its plane cuts the quadric.

Similarly it can be shown that if a point-pair is apolar to a quadric, its members are conjugate with regard to the quadric. For a point-double, the condition is that the point lie on the quadric.

Now it is clear that every quadric $(w)$ whose coördinates satisfy the linear equations

$$
\sum_{i, j=1}^{4} a_{i j}^{(k)} w_{i j}=0 \quad(k=1, \cdots, r)
$$

s apolar to each of the quadrics

$$
\sum_{i, j=1}^{4}\left(\lambda_{1} a_{i j}^{(1)}+\cdots+\lambda_{r} a_{i j}^{(r)}\right) x_{i} x_{j}=0 .
$$

This system we shall call the basal system of the linear system of quadrics $(w)$ defined by (13).

We shall denote the system of $\infty^{r}$ conics (embedded in $\infty^{r+1}$ quadrics) defined by a set of linear equations (13) of which $8-r$ are linearly independent, by $L_{r}$, and we now proceed to study the systems $L_{r}$ from $r=7$ to $r=0$. 
1. The $L_{7}$ or seven-parameter family is defined by one equation

$$
\sum_{i, j=1}^{4} a_{i j} w_{i j}=0 .
$$

Then the basal system consists of a single quadric

$$
\sum_{i, j=1}^{4} a_{i j} x_{i} x_{j}=0
$$

and every conic of the family is apolar to this quadric.

We have then the result: In the general $L_{7}$, the conics that lie in any plane are those which are apolar to a fixed conic in the plane, this fixed conic being the intersection of the plane with the base quadric (16). The system contains degenerate conics; in fact, the point-pairs of the general $L_{7}$ are $\infty^{5}$ in number. An arbitrary point of space may be associated with any one of the $\infty^{2}$ points of its polar plane in the base quadric to form a point-pair of the $L_{7}$. The point-doubles of the system consist of the points of the base quadric itself.

The only planes, in general, in which the configuration is projectively different from that of the general plane are those tangent to the base quadric. In such a plane the base quadric cuts a pair of lines; and these are conjugate with regard to every conic of the $L_{7}$ in this p'ane.

Special types of $L_{7}$.

(A) The base quadric is a cone.

The system has then the following distinctive features:

(a) the $\infty^{2}$ special planes pass through the vertex; $(b)$ there are $\infty^{1}$ of them, the tangents to the cone, in which all the $\infty^{4}$ conics of the $L_{7}$ touch a fixed line; $(c)$ the vertex of the cone is singular, in that it can be associated with any point of space to form a point-pair of the $L_{7}$.

$(B)$ The base quadric is a plane-pair.

In this case we have singular planes; namely the $L_{7}$ includes every conic in either of the planes of the basal plane-pair.

Conversely, if an $L_{7}$ includes all the conics of any plane, its basal quadrie must be a plane-pair of which this plane is one member.

$(C)$ The base quadric is a plane-double. Then (15) is of the form

$$
\sum_{i, j=1}^{4} d_{i} d_{j} w_{i j}=0
$$

We have studied this system $(\S 3,2)$ and have seen that it defines the quadrics that touch the plane $(d)$; in particular, then, the conics of the system are those that touch $(d)$, including those that lie in this plane. In $S_{9}$ this equation represents a hypersurface tangent to $\Sigma$ at all points of a $S_{5}$. 
2. The $L_{6}$, or 6-parameter family, is defined by two distinct equations

$$
\sum_{i, j=1}^{4} a_{i j} w_{i j}=0, \quad \sum_{i, j=1}^{4} b_{i j} w_{i j}=0 .
$$

The basal system consists of the pencil of quadrics

$$
\sum_{i, j=1}^{4}\left(\lambda a_{i j}+\mu b_{i j}\right) x_{i} x_{j}=0 .
$$

These have, in general, a common quartic curve $C_{4}$. Conversely, any conic apolar to two quadrics of the system (21) is apolar to all, and hence belongs to $L_{6}$.

The conics of the $L_{6}$ in an arbitrary plane are $\infty^{3}$ in number, and are apolar to all the conics of the pencil in which the basal system cuts the plane. The latter conics pass through the four points $P_{1}, P_{2}, P_{3}, P_{4}$, in which $C_{4}$ intersects the plane; and among them are in general three line-pairs. Each of these is a conjugate pair with regard to any conic of the $L_{6}$. Conversely, if a conic has two of these as conjugate line-pairs, then it is apolar to two distinct quadrics of the basal system, and hence belongs to the $L_{6}$.

This gives us the following

Theorem. Let a plane $(\pi)$ cut $C$ in the four points $P_{1}, P_{2}, P_{3}, P_{4}$. Then the conics of $L_{6}$ that lie in $(\pi)$ are those that have these points as a "polar quadrangle." (Cf. Reye, Geometrie der Lage, 4th ed., vol. 1, p. $261 \mathrm{ff}$.

Degenerate conics of the $L_{6}$. The point-doubles are necessarily common to all the basal quadrics, and hence are the points of the $C_{4}$. The members of a point-pair must be conjugate with regard to each of the basal quadrics. It is known that the polar planes of a point $P$ with regard to a pencil of quadrics meet in a line $l_{p}$. Hence $P$ and any point of this line form a point-pair of the $L_{6}$.

There are, however, four points which are exceptional to the above statement, namely the vertices of the cones of the basal pencil. We have then the result:

Theorem. The point-doubles of $L_{6}$ are the $\infty^{1}$ points of the curve $C_{4}$ common to the basal quadrics. There are four of them in an arbitrary plane (cf. $\$ 3$ at end). An arbitrary point determines a line, any point of which may be associated with it to form a point-pair of the $L_{6}$. But there are four singular points, the vertices of the cones of the basal system, each of which can be so associated with any point of the plane of the other three.

We have seen $(\S 3)$ that the point-pairs whose members lie on a given line are represented in $S_{9}$ by a $S_{2}^{1}$; hence any $S_{7}$ contains one of them. Hence the

TheoRem. The $L_{6}$ includes one point-pair whose members lie on an arbitrary line. 
Special types of $L_{6}$. In the foregoing, we have tacitly assumed that the pencil of basal quadrics is of the most general type. It is clear that the classification of $L_{6}$ 's is the same as that of pencils of quadrics. The latter is well known, and we shall not give it here.

We observe, however, that the first important specialization occurs when the pencil includes a plane-pair. In this case we may define the basal system by means of the plane-pair and any other member. Then the $L_{6}$ is the totality of members of an $L_{7}$ with regard to which two fixed planes are conjugate.

Similarly we get other special types. In particular, the totality of conics that touch two fixed planes form a special $L_{6}$.

Theorem. An $L_{6}$ can be such as to include all the conics of one plane, but not of more than one plane.

For consider the $L_{6}$ whose basal system is defined by two plane-pairs having a common plane; every conic in this plane belongs to the $L_{6}$. To prove the second part of the theorem, we note that an $L_{6}$ can be defined as the totality of conics common to two $L_{7}$ 's. But there is one and only one $L_{7}$ that contains all the conics of two planes, namely the one whose basal quadric is this planepair. Hence there is no $L_{6}$ that includes all these conics.

3. The $L_{5}$ is defined by three independent equations

$$
\sum_{i, j=1}^{4} a_{i j} w_{i j}=0, \quad \sum_{i, j=1}^{4} b_{i j} w_{i j}=0, \quad \sum_{i, j=1}^{4} c_{i j} w_{i j}=0 .
$$

The basal system is a net or linear two-parameter system represented by

$$
\sum_{i, j=1}^{4}\left(\lambda a_{i j}+\mu b_{i j}+\nu c_{i j}\right) x_{i} x_{j}=0,
$$

and the $L_{5}$ consists of all the conics apolar to every member of this system.

The basal system cuts an arbitrary plane in a so-called "bundle" or linear two-parameter family of conics. The $\infty^{2}$ conics of the $L_{5}$ in this plane are all apolar to the conics of the bundle, and they comprise a linear family in tangential coördinates. Reye (loc. cit.) calls such a family a "Schaarschaar." The nature of these two systems is well known. The conics of a bundle have in general no common point, those of a "Schaarschaar" no common tangent. A special case consists of the conics that have three common points in the one case, or in the other have three common tangents.*

Degenerate conics of the $L_{5}$. Clearly the point-doubles are eight in number, being the points common to all the base-quadrics. Thus we have (cf. §3) the

Theorem. The point-doubles of space correspond to points of a $S_{3}^{8}$ lying on $\Sigma$.

\footnotetext{
* For further treatment see Encyklopädie, III C 1, § 81 .
} 
There are also $\infty^{1}$ singular points, each of which may be associated with any point of a line to form a point-pair of the system. These singular points are the vertices of the cones of the basal system, and Reye (loc. cit.) shows that they lie on a sextic curve called the "Kerncurve" of the bundle. This curve has been considerably studied by Reye, and also, as an adjunct to a net of quadrics, by Sturm.*

Special types of $L_{5}$. There are a large number of special cases, and as before we shall not classify them. In general the basal net contains no plane-pair nor plane-double. If it contains one or more of either, the $L_{5}$ is correspondingly specialized. In particular, the conics that touch three fixed planes form a special $L_{5}$.

Theorem. An $L_{5}$ can contain all the conics of a plane. In this case it contains no other conics, and the conics of the $L_{5}$ in an arbitrary plane are the point-pairs on the line in which it intersects the plane of the $L_{5}$.

4. The $L_{4}$ is a system of conics determined by four independent linear equations

$$
\begin{aligned}
\sum_{i, j=1}^{4} a_{i j} w_{i j} & =0, & \sum_{i, j=1}^{4} c_{i j} w_{i j} & =0, \\
\sum_{i, j=1}^{4} b_{i j} w_{i j} & =0, & \sum_{i, j=1}^{4} d_{i j} w_{i j} & =0 .
\end{aligned}
$$

The basal system of quadrics is given by

$$
\sum_{i, j=1}^{4}\left(\lambda_{1} a_{i j}+\lambda_{2} b_{i j}+\lambda_{3} c_{i j}+\lambda_{4} d_{i j}\right) x_{i} x_{j}=0 .
$$

This system is called in German a Gebüsch. Its $\infty^{3}$ members have in general no common point. They include $\infty^{2}$ cones, and in general ten plane-pairs. $\dagger$

The Gebüsch being completely defined by any four of its members that are linearly independent, we may define it by means of four of its ten planepairs. If four plane-pairs be chosen arbitrarily, there is a Gebüsch linearly dependent on them. Hence we may define the $L_{4}$ as follows:

ThEOREM. The general $L_{4}$ has the property that there are ten pairs of planes, each a conjugate pair with regard to every conic of the $L_{4}$. Given four arbitrary pairs of planes, the totality of conics with regard to which each pair are conjugate constitute an $L_{4}$ of general type; and there are six additional pairs of planes, each pair conjugate with regard to all the conics of the system.

Degenerate conics. In general the $L_{4}$ has no point-doubles. It has $\infty^{2}$ point-pairs. To determine the order of the surface on which they lie, we recall $(\$ 3)$ that the point-pairs with one member on a given line are repre-

* See Encyklopädie, III C 2, p. 248, note 543.

$\dagger$ For proof see Reye, loc. cit., $\S 6$. 
sented in $S_{9}$ by a $S_{4}^{4}$. Hence in an arbitrary $S_{5}$ there are four points of this locus, and so in the general $L_{4}$ there are four point-pairs of which one member lies on an arbitrary line. Hence the points of the point-pairs lie on a quartic surface $\Phi_{4}$.

THEOREM. The surface $\Phi_{4}$ whose existence has just been established $i_{3}$ the locus of vertices of the $\infty^{2}$ cones of the basal system.

We observe that the $L_{4}$ has singular planes, in each of which there are $\infty^{2}$ conics instead of $\infty^{1}$. Namely, in any plane of the basal plane-pairs the conics of the $L_{4}$ form a Schaarschaar of the type considered in 3 .

Of course there are numerous special types of $L_{4}$. In particular, the conics that touch four fixed planes form a special $L_{4}$. Thus we have the following corollary to an earlier theorem:

The conics that touch four fixed planes form a system $L_{4}$, and there exist six other pairs of planes each conjugate with regard to every conic of the $L_{4}$.

5 . The $L_{3}$, or three parameter system, is defined by five independent equations:

$$
\sum_{i, j=1}^{4} a_{i j}^{(k)} w_{i j}=0 \quad(k=1, \cdots, 5) .
$$

These equations define $\infty^{4}$ envelope-quadrics, each apolar to the $\infty^{4}$ pointquadrics of the basal system

$$
\sum_{i, j=1}^{4}\left(\sum_{k=1}^{5} \lambda_{k} a_{i j}\right) x_{i} x_{j}=0 .
$$

There is complete duality between these two linear systems.* The former includes $\infty^{3}$ conics, which comprise the $L_{3}$, and the latter includes a dual system of $\infty^{3}$ cones.

We know that the conditions that a quadric be a conic lying in a given plane $(p)$ are the four linear equations

$$
\begin{array}{lll}
\sum_{i=1}^{4} w_{1 i} p_{i}=0, & \sum_{i=1}^{4} w_{3 i} p_{i}=0, \\
\sum_{i=1}^{4} w_{2 i} p_{i}=0, & \sum_{i=1}^{4} w_{4 i} p_{i}=0 .
\end{array}
$$

These, together with the five equations (20), form a system that has in general one and essentially only one solution; hence the $L_{3}$ has one conic in an arbitrary plane.

Theonem. The conics of the $L_{3}$ that lie in planes through a fixed line generate a surface of order 8, having the fixed line as a sextuple line.

Degenerate conics of the $L_{3}$. We may state the

* Cf. Reye, loc. cit., § 7 . 
Theorem. The point-pairs of the $L_{3}$ are $\infty^{1}$ in number, and lie on a curve $C_{10}$ of order ten. Their axes generate a ruled surface $K_{10}$ of order ten. Dually, the basal system includes $\infty^{1}$ plane-pairs, which envelope a developable $\Gamma_{10}$ of class ten; their lines of intersection lie on a ruled surface $\Phi_{10}$ of order ten.

Reye (loc. cit., $\S 7$ ) gives a number of very interesting relations among these four figures.

6. The $L_{2}$, or two-parameter linear system, is defined by a set of six independent equations. The totality of sets of solutions of such a system are linearly dependent on four, and define $\infty^{3}$ envelope-quadrics, which include the conics of the $L_{2}$.

Hence we have as alternative definition of the $L_{2}$ the equations

$$
\begin{gathered}
w_{i j}=\lambda_{1} a_{i j}+\lambda_{2} b_{i j}+\lambda_{3} c_{i j}+\lambda_{4} d_{i j} \quad(i, j=1, \cdots, 4), \\
\Delta(w)=0 .
\end{gathered}
$$

The system of quadrics (23) is dual to the Gebüsch discussed in 4; and the basal system is dual to the system of envelope-quadrics there discussed. We have then at once the following facts:

The conics of $L_{2}$ lie in $\infty^{2}$ planes, one in each plane in general, and these planes envelope a surface $\Phi_{4}$ of class four.

The $L_{2}$ includes in general ten point-pairs; their axes all lie on $\Phi_{4}$. The basal system contains $\infty^{2}$ plane-pairs, whose planes envelope the surface $\Phi_{4}$. In other words, the planes of these plane-pairs are the planes that contain conics of the $L_{2}$. Since they can be associated into pairs that belong to the basal system, we have the

THEOREM. The planes of the conics of the $L_{2}$ can be associated in pairs in such a way that the members of each pair are mutually conjugate with regard to every conic of the $L_{2}$.

The general $S_{3}$ in $S_{9}$ meets in eight points the $S_{6}^{8}$ which represents the conics through an arbitrary point; hence the

TheOREM. The $L_{2}$ includes 8 conics through an arbitrary point of space.

Special types of $L_{2}$. As in the earlier cases, when the basal system contains any plane-doubles, the conics of $L_{2}$ all touch every such plane; and in particular, the conics that touch six fixed planes form an $L_{2}$ of very special type. The dual system (viz., the Gebüsch of quadrics through six fixed points) has been somewhat studied.*

In general, the $L_{2}$ will have no singular plane containing more than one conic. There may, however, be such planes. There are four types, of which one reduces to a complete $S_{3}$, and the others are incomplete $L_{2}$ 's with singular planes. Namely:

* Cf. Encyklopädie, III C 2, § 141. 
(1) One singular plane containing $\infty^{1}$ conics.

(2) Two singular planes, each containing $\infty^{1}$ conics.

(3) One singular plane containing $\infty^{2}$ conics.

(4) If all four conics are coplanar, every linear combination of them is a conic, and we have a complete three-parameter linear system of conics in the plane in question.

7. The $L_{1}$, or one-parameter linear system, is most conveniently defined as a system linearly dependent on three sets of numbers:

$$
\begin{gathered}
w_{i j}=\lambda_{1} a_{i j}+\lambda_{2}, b_{i j}+\lambda_{3} c_{i j}, \\
\Delta(w)=0 .
\end{gathered}
$$

Theorem. The planes of the conics of a general $L_{1}$ form a developable of class 6.

For the conics whose planes pass through a given point are represented in $S_{9}$ by a $S_{7}^{6}(\S 3)$ and hence an arbitrary $S_{2}$ contains 6 of them. We find easily also the following results.

TheOREM. The conics of the general $L_{1}$ lie on a surface of order eight.

TheOREM. There are four conics of the $L_{1}$ that touch an arbitrary plane.

CoRollary. The conics of the $L_{1}$ cut a given plane in points of a curve of order 8. They determine on this curve an involution of points, in which four pairs of points are self-corresponding.

The basal system of the $L_{1}$ consists of a linear $\infty^{6}$ of point-quadrics. We know (2) that such a system includes eight plane-doubles. But every envelope-quadric touches every plane that is a plane-double apolar to it; so that every conic of the $L_{1}$ touches these eight fixed planes. This leads to another definition of the $L_{1}$.

Theorem. The general $L_{1}$ consists of the totality of conics that touch eight fixed planes, whereof seven may be chosen arbitrarily.

In other words: Let seven planes be chosen arbitrarily in space. There are $\infty^{1}$ planes, generating a sextic developable, in each of which the seven lines cut by the fixed planes are all tangent to a conic. The conics thus determined lie on a surface of order eight, and all touch an eighth fixed plane.

8. The $L_{0}$, or system of conics whose coördinates-satisfy eight linear equations, may be defined by

$$
w_{i j}=\lambda_{1} a_{i j}+\lambda_{2} b_{i j} \quad \Delta(w)=0 .
$$

We may state the result:

The general $L_{0}$ consists of four conics having common tangent planes that generate a quartic developable. A system of this kind is uniquely determined by any two quadrics; the planes of the four conics form the only tetrahedron that is self-conjugate with regard to all the quadrics of the series containing the $L_{0}$. 


\section{Complete Linear Systems}

We now propose to determine all possible types of complete linear systems. First we will note that such a system may be given parametrical!y in the form

$$
w_{i j}=\lambda_{1} a_{i j}^{(1)}+\cdots+\lambda_{7} a_{i j}^{(r)} \quad(i, j=1, \cdots, 4),
$$

where $\Delta(w) \equiv 0$ for all values of $\left(\lambda_{1}, \cdots, \lambda_{7}\right)$.

Consider, in particular, two conics $(a)$ and $(b)$ of a complete linear system. Then every quadric

$$
w_{i j}=\lambda a_{i j}+\mu b_{i j}
$$

must be a conic of the system. We have then

$$
\lambda^{4} \Delta(a)+\lambda^{3} \mu \theta(a, b)+\lambda^{2} \mu^{2} \Phi(a, b)+\lambda \mu^{3} \theta(b, a)+\mu^{4} \Delta(b)=0 .
$$

We have already, since $(a)$ and $(b)$ are conics,

$$
\Delta(a)=\Delta(b)=0,
$$

and the following further relations are necessary:

$$
\Theta(a, b)=0, \quad \Theta(b, a)=0, \quad \Phi(a, b)=0 .
$$

From the results of $\S 5$, it follows that we must have one of the following situations:

(A) If neither $(a)$ nor $(b)$ is degenerate, then (1) they are coplanar; (2) each touches the plane of the other, and they cut the line of intersection of their planes in a harmonic set of points.

$(B)$ When $(a)$ is a proper conic, and $(b)$ is degenerate, then (1) if $(b)$ is a point-pair, its axis meets $(a)$, and the plane of $(a)$ touches $(b) ;(2)$ if (b) is a point-double, the plane of $(a)$ touches it.

(C) When both conics are degenerate, then (1) if both are point-pairs, their axes intersect; (2) if either is a point-double, the equation is satisfied.

Considering these cases, we see that except in $A 2$ and $B 1$, the conics are coplanar. In case $A 2$ they are necessarily tangent; and in case $B 1$, either they are coplanar, or the point-pair $(b)$ has one member lying on $(a)$.

We introduce now the following

Definition. A point-pair shall be said to be tangent to a proper conic, when one of its points lies on the conic.

And we have the result: Every two conics of a complete linear system are either coplanar or tangent. Conversely, we see without difficulty that if two conics are coplanar or tangent, every quadric linearly dependent on them is a conic.

Concerning the locus of these conics, we present without detailed proof the following statement:

Theorem. If $(w)=\lambda(a)+\mu(b)$ be a one-parameter complete linear system, it is of one of the two types: 
(1) the SERIES (Schaar) consisting of the conics in a plane that touch four fixed lines in the plane;

(2) the one-parameter SHEAF, consisting of the conics cut from a quadric cone by the planes of a pencil whose axis is a tangent line to the cone. The axis of the pencil, which is evidently tangent to all the conics of the sheaf, we shall call the axis of the sheaf; and the point of contact we shall call the vertex of the sheaf.

We may divide complete linear systems in general into two classes, according as all their members lie in the same plane or not. The planar systems we shall not discuss further than to remark that they are the systems studied in plane geometry as linear systems in tangential coördinates.

Let us consider a system in which not all the conics are coplanar. By brief reasoning, which we here suppress, one arrives at this result:

THEOREM. In any non-planar complete linear system, the members all touch a fixed line at a fixed point; and conversely, any number of conics tangent to a fixed line at a fixed point will determine a complete linear system.

We have not considered degenerate conics. Evidently a point-pair can belong to a system of this type if and only if it has either (1) one member at the vertex, or (2) both members on the axis; and a point-double can belong to the system, if and only if it lies on the axis.

Definition. A non-planar linear complete system, of which the coördinates of the members are linearly dependent on those of $r+1$ linearly independent conics, shall be called an r-parameter sheaf.

We now examine the most general sheaf and find readily the

THEOREM. The most general sheaf of conics consists of the totality of conics that touch a fixed axis at a fixed point. It is projectively equivalent to the system whose coördinates are given by the matrix

$$
\left[\begin{array}{cccc}
0 & 0 & 0 & a_{1} \\
0 & 0 & 0 & a_{2} \\
0 & 0 & a_{0} & a_{3} \\
a_{1} & a_{2} & a_{3} & a_{4}
\end{array}\right]
$$

for all sets of values $\left(a_{0}, a_{1}, a_{2}, a_{3}, a_{4}\right)$ not all zero. It follows that such $a$ sheaf is a four-parameter system.

Definition. A four-parameter sheaf shall be called an entire sheaf.

We note the following properties of the entire sheaf:

An entire sheaf is determined by a point and a line through it, hence there are in all $\infty^{5}$ of them.

Any proper conic is a member of $\infty^{1}$ entire sheaves, one with its vertex at each point of the conic. 
If two entire sheaves have in common a proper conic, their axes are coplanar, and they have in common a pencil of conics having double contact.

A point-pair belongs to $\infty^{2}$ entire sheaves, viz., to any one whose vertex is at either point of the pair, or whose axis is the axis of the pair.

Every two entire sheaves have in common one point-pair, made up of the vertices of the sheaves. Two entire sheaves have more than one common point-pair, if they have the same vertex, or if the vertex of one passes through the axis of the other.

A point-double belongs to every entire sheaf whose axis passes through the point.

Translating these results in $S_{9}$, we will denote by $\Lambda$ the singular domain $S_{6}^{10}$ that represents point-pairs, and by $\Phi$ the linear $S_{4}$ on $\Sigma$ that represents an entire sheaf.

Then $\Sigma$ contains $\infty^{5} \Phi$ 's. Through an ordinary point of $\Sigma$ there are $\infty^{1}$, of which any two intersect in a line. In general, two $\Phi$ 's do not intersect at any ordinary point of $\Sigma$, but at a single point of $\Lambda$. If they intersect at any ordinary point, they have a common line.

Through an arbitrary point of $\Lambda$, there are in general $\infty^{2} \Phi$ 's. Through any point of the $S_{3}^{8}$ that represents point-doubles there are $\infty^{3} \Phi$ 's.

The point-pairs of the above entire sheaf are determined by either $a_{0}=0$, or $a_{1}=a_{2}=0$, corresponding respectively to point-pairs having one member at the vertex, and to those having both members on the axis. The former are represented in $\Phi$ by a linear three-space $Q$, the latter by a plane $R$.

The point-doubles are determined by $a_{1}=a_{2}=0, a_{0} a_{4}=a_{3}^{2}$, and are represented in $\Phi$ by a $S_{1}^{2}$, i. e., a conic.

The entire sheaf contains sheaves of one, two, and three parameters, which we denote by $T_{1}, T_{2}, T_{3}$ respectively, with reference to which we give here only summary statements.

1. The one parameter sheaf $T_{1}$ we have already considered. If $(a)$ and $(b)$ be any two conics of $T_{4}$, then a $T_{1}$ is given by

$$
w_{i}=\lambda a_{i}+\mu b_{i} \quad(i=0, \cdots, 4) .
$$

This corresponds to a line in $S_{4}$. There are therefore $\infty^{6} T_{1}$ 's in any $T_{4}$.

If $(a)$ and $(b)$ are not coplanar, the conics lie on a cone that touches the axis at the vertex of $T$. In this case the sheaf contains just one degenerate conic. There are, however, special cases not described here.

2. The $T_{2}$ is defined by the equations

$$
w_{i}=\lambda_{1} a_{i}+\lambda_{2} b_{i}+\lambda_{3} c_{i} \quad(i=0,1,2,3,4),
$$

where $(a),(b)$, and $(c)$ are linearly independent members of a $T_{4}$. It is represented in $S_{4}$ by a plane $(\pi)$.

Trans. Am. Math. Soc. '25 
The plane $(\pi)$ cuts $Q$ in a line $k$ and $R$ in a point $K$, and can be described by lines drawn from $K$ to the points of $k$. Each of these lines represents a planar $T_{1}$, as may easily be seen. Hence the $T_{2}$ may be regarded as composed of $\infty^{1}$ planar $T_{1}$ 's. Moreover, the common tangents to the members of each planar $T_{1}$ meet the axis at the same points $R_{1}, R_{2}$, since each of the lines cuts $R$ at the same point. We have then the following definition of the $T_{2}$ :

Theorem. The totality of conics of a $T_{4}$ that touch two fixed planes, whose intersection does not meet the axis of the $T_{4}$, constitute a $T_{2}$ of the most general type.

Special types of $T_{2}$ correspond to special relations of $(\pi)$ to the space containing the line $k$ and the plane containing the point $K$. For example, it may happen that $K$ lies on $k$. In this case $a T_{2}$ of the type under consideration consists of all the conics of a $T_{4}$ that have contact of the second order with one another at the vertex, and also touch a fixed plane not through the vertex.

Two other special types are evidently those in which either $k$ or $K$ is indeterminate. The latter is a planar $T_{2}$, and we find the

Theorem. The general planar $T_{2}$ consists of all the conics of a $T_{4}$ that lie in a plane and touch any two lines so drawn from two fixed points on the axis of the $T_{4}$ as to intersect on a fixed line in the plane. It follows that the common tangents to any two conics of the system intersect on this fixed line.

3. The three-parameter system $T_{3}$ can be treated in the same way. A $T_{3}$ is represented in $S_{4}$ by a space $S_{3}$; and in general this cuts $Q$ in a plane $N$, and $R$ in a line $n$. To the former correspond $\infty^{2}$ point-pairs of the $T_{3}$, consisting of the vertex $P$, taken with the $\infty^{2}$ points of a plane $(\sigma)$. To the latter correspond $\infty^{1}$ point-pairs on the axis of the $T$.

The $S_{3}$ may be regarded as swept out by a pencil of planes through the line $n$. Each of these planes corresponds to a planar $T_{2}$ of the kind just studied. We have seen that such a system has the property that the common tangents to any two of its members meet on a fixed line $l$. In our pencil of $T_{2}$ 's, these fixed lines all lie in the plane $(\sigma)$. Also the vertex of the cone of any $T_{1}$ in the $T_{3}$ lies on $(\sigma)$. We may then define the $T_{3}$ as follows:

Let a plane $(\sigma)$ and a point-pair $\left(R_{1}, R_{2}\right)$ on the axis be chosen arbitrarily. The totality of conics of the $T_{4}$, which touch a pair of lines drawn from $R_{1}$ and $R_{2}$ to any point of the plane $(\sigma)$, generaie $a T_{3}$. The common tangents to any two coplanar members of the $T_{3}$ meet on $(\sigma)$, and the vertex of the cone through any two other members lies on $(\sigma)$.

We mention two special types. There is one special $T_{3}$, namely, $a_{0}=0$, consisting of all the point-pairs whereof the vertex is one member. Another type is represented by a $S_{3}$ through the plane containing the point $K$. It is obvious that such a $S_{3}$ represents a planar $T_{3}$.

Cambridge, Mass., June, 1913. 